\title{
MAGNETOHYDRODYNAMIC APPROACH TO SOLVABILITY OF THE THREE-DIMENSIONAL STATIONARY EULER EQUATIONS
}

\author{
TAKAHIRO NISHIYAMA \\ Department of Mechanical Engineering, Fukuoka Institute of Technology, Fukuoka 811-0295, Japan \\ e-mail:nisiyama@fit.ac.jp
}

(Received 11 December, 2000; accepted 23 August, 2001)

\begin{abstract}
A sequence of solutions to the Galerkin approximation of a nonstationary magnetohydrodynamic system is proved to converge to a measure-valued solution, in the sense of R. J. DiPerna-A. J. Majda, to the three-dimensional stationary Euler equations.
\end{abstract}

2000 Mathematics Subject Classification. Primary 35Q30. Secondary 35D05, $76 \mathrm{~W} 05$.

1. Introduction. Let $\boldsymbol{u}\left(: \Omega \rightarrow \mathbf{R}^{3}\right)$ and $p(: \Omega \rightarrow \mathbf{R})$ be the velocity and pressure, respectively, of a steady-state inviscid incompressible fluid with unit density in a three-dimensional domain $\Omega$. They satisfy the stationary Euler equations

$$
\boldsymbol{u} \cdot \nabla \boldsymbol{u}=-\nabla p, \quad \nabla \cdot \boldsymbol{u}=0 .
$$

In the two-dimensional and the axisymmetric cases, the existence of a solution to (1) with nonvanishing vorticity has been investigated by variational methods in, for example, $[\mathbf{2}, \mathbf{3}, \mathbf{5}, \mathbf{9}, \mathbf{1 1}, \mathbf{1 6}, \mathbf{1 9}, \mathbf{2 0}]$ and by some other methods in $[\mathbf{1 7}, \mathbf{1 8}, \mathbf{2 2}]$. On the other hand, in the three-dimensional case, these methods seem inapplicable and, as far as the author knows, the existence has not been rigorously proved, except under a special situation accompanied with a leakage in [1] or under the condition that $\boldsymbol{u}$ and $\boldsymbol{\nabla} \times \boldsymbol{u}$ are parallel in $[\mathbf{4 , 1 2 , 2 3 ]}$.

To obtain solutions to (1), Moffatt [14] proposed the nonstationary equations for a viscous and perfectly conductive magneto-fluid:

$$
\left.\begin{array}{c}
\boldsymbol{v}_{t}+\boldsymbol{v} \cdot \nabla \boldsymbol{v}=-\nabla q+\boldsymbol{B} \cdot \nabla \boldsymbol{B}-\nabla\left(|\boldsymbol{B}|^{2} / 2\right)+\Delta \boldsymbol{v}, \\
\boldsymbol{B}_{t}+\boldsymbol{v} \cdot \nabla \boldsymbol{B}=\boldsymbol{B} \cdot \nabla \boldsymbol{v}, \\
\boldsymbol{\nabla} \cdot \boldsymbol{v}=\boldsymbol{\nabla} \cdot \boldsymbol{B}=0 .
\end{array}\right\}
$$

Here the density and viscosity are equal to unit and $(\boldsymbol{v}, \boldsymbol{B}, q): \Omega \times\{t>0\} \rightarrow$ $\mathbf{R}^{3} \times \mathbf{R}^{3} \times \mathbf{R}$ stand for the velocity, magnetic field and pressure, respectively. He asserted the relaxation of (2) (with appropriate initial data) to an equilibrium

$$
\boldsymbol{v}=\mathbf{0}, \quad \boldsymbol{B} \cdot \boldsymbol{\nabla} \boldsymbol{B}=\nabla\left(q+|\boldsymbol{B}|^{2} / 2\right), \quad \nabla \cdot \boldsymbol{B}=0,
$$

as the time $t \rightarrow \infty$, which means that $\boldsymbol{u}=\left.\boldsymbol{B}\right|_{t=\infty}$ is a solution to (1) with $p=-\left.\left(q+|\boldsymbol{B}|^{2} / 2\right)\right|_{t=\infty}$. This is worthy of remark. However, to justify it rigorously, 
we need the existence of a temporally global solution to (2) in the sense of distribution in space at the weakest. In fact, the existence seems difficult to prove because of the perfect conductivity of the magneto-fluid. Moreover, even if it is proved and the decay $\boldsymbol{v} \rightarrow \mathbf{0}$ (as $t \rightarrow \infty)$ in $\boldsymbol{L}^{2}(\Omega)\left(=\left(L^{2}(\Omega)\right)^{3}\right)$ is obtained, we cannot derive $\boldsymbol{v}_{t} \rightarrow \mathbf{0}$ in $L^{2}(\Omega)$ as easily as Moffatt asserted. Moffatt [15] considered the relaxation of another magnetohydrodynamic system which has the same mathematical difficulty.

In [21], Vallis et al. introduced the nonstationary system of equations for an inviscid and perfectly conductive magneto-fluid with artificial terms:

$$
\left.\begin{array}{c}
\boldsymbol{v}_{t}+\boldsymbol{v} \cdot \nabla \boldsymbol{v}=-\nabla q+\boldsymbol{B} \cdot \nabla \boldsymbol{B}-\boldsymbol{\nabla}\left(|\boldsymbol{B}|^{2} / 2\right)+\alpha \boldsymbol{v}_{t} \times(\boldsymbol{\nabla} \times \boldsymbol{v}), \\
\boldsymbol{B}_{t}+\left(\boldsymbol{v}+\alpha \boldsymbol{v}_{t}\right) \cdot \boldsymbol{\nabla} \boldsymbol{B}=\boldsymbol{B} \cdot \boldsymbol{\nabla}\left(\boldsymbol{v}+\alpha \boldsymbol{v}_{t}\right), \\
\boldsymbol{\nabla} \cdot \boldsymbol{v}=\boldsymbol{\nabla} \cdot \boldsymbol{B}=0 .
\end{array}\right\}
$$

Here $\alpha$ is a nonzero constant. They asserted the relaxation of (3) to a steady state as $t \rightarrow \infty$. However, it is also difficult to prove rigorously. In fact, even if the smooth solvability is assumed globally in time, we do not know whether $\boldsymbol{B}_{t} \rightarrow \mathbf{0}$ as $t \rightarrow \infty$.

Combining the ideas of Moffatt and Vallis et al., we introduce

$$
\left.\begin{array}{c}
\boldsymbol{v}_{t}+\boldsymbol{v} \cdot \nabla \boldsymbol{v}=-\nabla q+\boldsymbol{B} \cdot \boldsymbol{\nabla} \boldsymbol{B}-\nabla\left(|\boldsymbol{B}|^{2} / 2\right)+\boldsymbol{v}_{t} \times(\nabla \times v)+\Delta \boldsymbol{v}, \\
\boldsymbol{B}_{t}+\left(\boldsymbol{v}+\boldsymbol{v}_{t}\right) \cdot \nabla \boldsymbol{B}=\boldsymbol{B} \cdot \boldsymbol{\nabla}\left(\boldsymbol{v}+\boldsymbol{v}_{t}\right), \\
\boldsymbol{\nabla} \cdot \boldsymbol{v}=\boldsymbol{\nabla} \cdot \boldsymbol{B}=0 .
\end{array}\right\}
$$

For this system, if we assume the temporally global and smooth solvability, which seems difficult to prove as well as for (2) and (3), then we can show that $\boldsymbol{v} \rightarrow \mathbf{0}$, $\boldsymbol{v}_{t} \rightarrow \mathbf{0}$ and $\boldsymbol{B}_{t} \rightarrow \mathbf{0}$ as $t \rightarrow \infty$.

The aim of this paper is to prove that a sequence of solutions to the Galerkin approximation of (4) converges to a measure-valued solution to (1) when we let $t$ and the number of basis functions go to infinity simultaneously. The concept of measure-valued solutions was applied to the nonstationary Euler equations by DiPerna and Majda [7,8]. They justified its utility as a tool for discussion of complex phenomena in Euler flows (see also [6]).

2. Preliminaries. Let us introduce our notation. We assume that $\Omega\left(\subset \mathbf{R}^{3}\right)$ is an open, bounded and simply connected domain whose boundary $\partial \Omega$ is sufficiently smooth. By $C_{0}^{\infty}(\Omega)$, we denote the set of all $\infty$-times continuously differentiable functions defined on $\Omega$ whose supports are compact. By $C_{0, \sigma}^{\infty}(\Omega)$, we mean the set of all three-dimensional divergence-free functions whose components belong to $C_{0}^{\infty}(\Omega)$. The product $((\cdot, \cdot))$ and the norm $\|\cdot\|$ are defined by

$$
((\boldsymbol{f}, \boldsymbol{g}))=\int_{\Omega} \boldsymbol{f}(\boldsymbol{x}) \cdot \boldsymbol{g}(\boldsymbol{x}) \mathrm{d} \boldsymbol{x} \quad \text { and } \quad\|\boldsymbol{f}\|=((\boldsymbol{f}, \boldsymbol{f}))^{1 / 2} .
$$

The spaces $\boldsymbol{H}_{\sigma}$ and $\boldsymbol{H}_{\sigma}^{1}$ represent the closures of $\boldsymbol{C}_{0, \sigma}^{\infty}(\Omega)$ with respect to $\|\cdot\|$ and $\|\cdot\|_{1}$, respectively, where $\|\cdot\|_{j}(j \in \mathbb{N})$ is the norm in the vector-valued Sobolev space of the $j$-th order denoted by $\boldsymbol{W}_{2}^{j}(\Omega)$.

By $M(G)$, we denote the space of Radon measures on $G=\Omega, \mathbf{R}^{3}$ or $S^{2}$, where $S^{2}$ stands for the unit sphere. The total variation $|\mu|_{M(G)}$ of each $\mu \in M(G)$ is given by the supremum of $\left|\int_{G} \phi \mathrm{d} \mu\right|$ for all continuous functions $\phi$ on $G$ such that the support 
of $\phi$ is compact and $|\phi| \leq 1$. By $M^{+}(G)$, we denote the subspace of nonnegative measures in $M(G)$. The subspace of measures with unit total variation in $M^{+}(G)$ is denoted by $\operatorname{Prob} M(G)$.

By $\boldsymbol{w}_{1}^{k} \in \boldsymbol{H}_{\sigma}^{1} \cap \boldsymbol{W}_{2}^{3}(\Omega)(k=1,2,3, \ldots)$, we denote eigenfunctions for the problem

$$
\Delta \boldsymbol{w}=\nabla s-\lambda \boldsymbol{w}, \quad \nabla \cdot \boldsymbol{w}=0,\left.\quad \boldsymbol{w}\right|_{\partial \Omega}=\mathbf{0}, \quad\|\boldsymbol{w}\|=1
$$

with functions $s=s_{k}$ and eigenvalues $\lambda=\lambda_{1, k}$ such that $\nabla s_{k} \in \boldsymbol{W}_{2}^{1}(\Omega), 0<\lambda_{1, k} \leq \lambda_{1, k+1}$ and $\lim _{k \rightarrow \infty} \lambda_{1, k}=\infty$ ([13, Chapter 2]). Then $\left\{\boldsymbol{w}_{1}^{k}\right\}_{k=1}^{\infty}$ is a complete orthonormal system in $\boldsymbol{H}_{\sigma}$.

By $\boldsymbol{w}_{2}^{k} \in \boldsymbol{H}_{\sigma} \cap \boldsymbol{W}_{2}^{1}(\Omega)(k=1,2,3, \ldots)$, we denote eigenfunctions for the problem

$$
\nabla \times \boldsymbol{w}=\lambda \boldsymbol{w},\left.\quad \boldsymbol{w} \cdot \boldsymbol{n}\right|_{\partial \Omega}=0, \quad\|\boldsymbol{w}\|=1
$$

with eigenvalues $\lambda=\lambda_{2, k}$ such that $0<\left|\lambda_{2, k}\right| \leq\left|\lambda_{2, k+1}\right|$ and $\lim _{k \rightarrow \infty}\left|\lambda_{2, k}\right|=\infty$ ([23]). Here $\boldsymbol{n}$ is the unit outward normal vector on $\partial \Omega$. Then $\left\{\boldsymbol{w}_{2}^{k}\right\}_{k=1}^{\infty}$ is a complete orthonormal system in $\boldsymbol{H}_{\sigma}$. It should be noted that const. $\boldsymbol{w}_{2}^{k}$ for an arbitrary $k$ satisfies (1). Indeed,

$$
\boldsymbol{w}_{2}^{k} \cdot \nabla \boldsymbol{w}_{2}^{k}=\left(\nabla \times \boldsymbol{w}_{2}^{k}\right) \times \boldsymbol{w}_{2}^{k}+\nabla\left(\left|\boldsymbol{w}_{2}^{k}\right|^{2} / 2\right)=\nabla\left(\left|\boldsymbol{w}_{2}^{k}\right|^{2} / 2\right)
$$

The reason for using $\left\{\boldsymbol{w}_{2}^{k}\right\}_{k=1}^{\infty}$ is that we can obtain (15) below.

For vectors $\boldsymbol{f}=\left(f_{1}, f_{2}, f_{3}\right)$ and $\boldsymbol{g}=\left(g_{1}, g_{2}, g_{3}\right)$, we denote the matrix whose $(i, j)$ component is equal to $f_{i} g_{j}$ by $\boldsymbol{f} \otimes \boldsymbol{g}$. By $\nabla \boldsymbol{f}$, we denote the Jacobian matrix of $\boldsymbol{f}(\boldsymbol{x})$. We define the product of $3 \times 3$-matrices $F=\left(f_{i j}\right)$ and $G=\left(g_{i j}\right)$ by $F: G=$ $\sum_{i=1}^{3} \sum_{j=1}^{3} f_{i j} g_{i j}$.

3. Result. Let us consider the system of equations for $\left\{a_{n, k}(t)\right\}_{k=1}^{n}$ and $\left\{b_{n, k}(t)\right\}_{k=1}^{n}$ with $n \in \mathbb{N}$ fixed:

$$
\begin{gathered}
\frac{\mathrm{d} a_{n, k}}{\mathrm{~d} t}+\sum_{j=1}^{n}\left(\left(\left(\nabla \times \boldsymbol{v}^{n}\right) \times \boldsymbol{w}_{1}^{j}, \boldsymbol{w}_{1}^{k}\right)\right) \frac{\mathrm{d} a_{n, j}}{\mathrm{~d} t}=\left(\left(\boldsymbol{B}^{n} \cdot \nabla \boldsymbol{B}^{n}-\boldsymbol{v}^{n} \cdot \nabla \boldsymbol{v}^{n}, \boldsymbol{w}_{1}^{k}\right)\right)-\lambda_{1, k} a_{n, k}, \\
\frac{\mathrm{d} b_{n, k}}{\mathrm{~d} t}=\left(\left(\boldsymbol{B}^{n} \cdot \nabla\left(\boldsymbol{v}^{n}+\boldsymbol{v}_{t}^{n}\right)-\left(\boldsymbol{v}^{n}+\boldsymbol{v}_{t}^{n}\right) \cdot \nabla \boldsymbol{B}^{n}, \boldsymbol{w}_{2}^{k}\right)\right)
\end{gathered}
$$

where

$$
\boldsymbol{v}^{n}=\sum_{j=1}^{n} a_{n, j}(t) \boldsymbol{w}_{1}^{j}, \quad \boldsymbol{B}^{n}=\sum_{j=1}^{n} b_{n, j}(t) \boldsymbol{w}_{2}^{j} .
$$

It is an approximation of (4) with the $2 n$ basis functions $\left\{\boldsymbol{w}_{1}^{k}\right\}_{k=1}^{n},\left\{\boldsymbol{w}_{2}^{k}\right\}_{k=1}^{n}$. Indeed, (5) can be rewritten as

$$
\left(\left(\boldsymbol{v}_{t}^{n}+\left(\nabla \times \boldsymbol{v}^{n}\right) \times \boldsymbol{v}_{t}^{n}, \boldsymbol{w}_{1}^{k}\right)\right)=\left(\left(\boldsymbol{B}^{n} \cdot \nabla \boldsymbol{B}^{n}-\boldsymbol{v}^{n} \cdot \nabla \boldsymbol{v}^{n}+\Delta \boldsymbol{v}^{n}, \boldsymbol{w}_{1}^{k}\right)\right) .
$$


The initial conditions to impose on (5) and (6) are

$$
a_{n, k}(0)=\left(\left(\boldsymbol{v}_{0}, \boldsymbol{w}_{1}^{k}\right)\right), \quad b_{n, k}(0)=\left(\left(\boldsymbol{B}_{0}, \boldsymbol{w}_{2}^{k}\right)\right),
$$

where $\boldsymbol{v}_{0} \in \boldsymbol{H}_{\sigma}^{1}$ and $\boldsymbol{B}_{0}(\not \equiv \mathbf{0}) \in \boldsymbol{H}_{\sigma}$ are arbitrary. The following is our main theorem.

THeOrem. The initial value problem (5)-(7) has a unique smooth solution globally in time. There exist sequences $\left\{n_{m} \in \mathbb{N} \mid n_{m}<n_{m+1}\right\}$ and $\left\{t_{m} \mid 0<t_{m}<t_{m+1}\right.$, $\left.\lim _{m \rightarrow \infty} t_{m}=\infty\right\}(m=1,2,3, \ldots)$ such that $\left\|\left.\boldsymbol{v}^{n_{m}}\right|_{t=t_{m}}\right\|,\left\|\nabla \times\left.\boldsymbol{v}^{n_{m}}\right|_{t=t_{m}}\right\|$ and $\left\|\left.\boldsymbol{v}_{t}^{n_{m}}\right|_{t=t_{m}}\right\|$ converge to zero and $\left.\left|\boldsymbol{B}^{n_{m}}\right|_{t=t_{m}}\right|^{2}$ converges weakly-* to a measure $\mu$ in $M(\Omega)$ satisfying

$$
|\mu|_{M(\Omega)} \leq\left\|\boldsymbol{v}_{0}\right\|^{2}+\left\|\nabla \times \boldsymbol{v}_{0}\right\|^{2}+\left\|B_{0}\right\|^{2} .
$$

In addition, if

$$
\sum_{j=1}^{\infty} \lambda_{2, j}^{-1}\left(\left(\boldsymbol{B}_{0}, \boldsymbol{w}_{2}^{j}\right)\right)^{2} \neq 0,
$$

then $|\mu|_{M(\Omega)}>0$ holds. As $m \rightarrow \infty$, the system (5)-(7) with $n=n_{m}$ and $t=t_{m}$ yields the existence of a $\mu$-measurable map $\boldsymbol{x}(\in \Omega) \mapsto\left\{v_{1, \boldsymbol{x}}, v_{2, \boldsymbol{x}}\right\}\left(\in M^{+}\left(\mathbf{R}^{3}\right) \oplus \operatorname{Prob} M\left(S^{2}\right)\right)$ such that $\left\{\mu, v_{1, x}, v_{2, x}\right\}$ is a measure-valued solution to (1) in DiPerna-Majda's sense, that is,

$$
\begin{gathered}
\int_{\Omega} \nabla \boldsymbol{\Phi}:\left(\int_{S^{2}} \frac{\boldsymbol{u}}{|\boldsymbol{u}|} \otimes \frac{\boldsymbol{u}}{|\boldsymbol{u}|} \mathrm{d} \nu_{2, \boldsymbol{x}}\right) \mathrm{d} \mu=0, \\
\int_{\Omega} \nabla \phi \cdot\left(\int_{\mathbf{R}^{3}} \frac{\boldsymbol{u}}{1+|\boldsymbol{u}|^{2}} \mathrm{~d} \nu_{1, \boldsymbol{x}}\right)(1+h) \mathrm{d} \boldsymbol{x}=0
\end{gathered}
$$

are satisfied for all $\mathbf{\Phi} \in \boldsymbol{C}_{0, \sigma}^{\infty}(\Omega)$ and all $\phi \in C_{0}^{\infty}(\Omega)$. Here $h$ is the Radon-Nikodym derivative of the absolutely continuous part of $\mu$ with respect to the Lebesgue measure.

Proof. Since the matrix whose $(k, j)$-component is given by $\left(\left(\left(\nabla \times \boldsymbol{v}^{n}\right) \times \boldsymbol{w}_{1}^{j}, \boldsymbol{w}_{1}^{k}\right)\right)$ is anti-symmetric and all its eigenvalues have zero real parts, the problem (5)-(7) has a unique smooth solution at least locally in time.

Multiplying (5) by $a_{n, k}$ or $(\mathrm{d} / \mathrm{d} t) a_{n, k}$, we get

$$
\begin{aligned}
\frac{1}{2} \frac{\mathrm{d}}{\mathrm{d} t}\left\|\boldsymbol{v}^{n}\right\|^{2} & =\left(\left(-\left(\boldsymbol{\nabla} \times \boldsymbol{v}^{n}\right) \times \boldsymbol{v}_{t}^{n}+\boldsymbol{B}^{n} \cdot \boldsymbol{\nabla} \boldsymbol{B}^{n}, \boldsymbol{v}^{n}\right)\right)-\left\|\nabla \times \boldsymbol{v}^{n}\right\|^{2}, \\
\left\|\boldsymbol{v}_{t}^{n}\right\|^{2} & =\left(\left(\boldsymbol{B}^{n} \cdot \boldsymbol{\nabla} \boldsymbol{B}^{n}-\boldsymbol{v}^{n} \cdot \nabla \boldsymbol{v}^{n}, \boldsymbol{v}_{t}^{n}\right)\right)-\frac{1}{2} \frac{\mathrm{d}}{\mathrm{d} t}\left\|\nabla \times \boldsymbol{v}^{n}\right\|^{2} .
\end{aligned}
$$

Here we noted that $((\boldsymbol{f} \cdot \nabla \boldsymbol{g}, \boldsymbol{h}))=-((\boldsymbol{f} \cdot \nabla \boldsymbol{h}, \boldsymbol{g}))(=0$ if $\boldsymbol{g}=\boldsymbol{h})$ holds for $\boldsymbol{f}, \boldsymbol{g}$ and $\boldsymbol{h}$ satisfying $\nabla \cdot \boldsymbol{f}=0$ and $\left.(\boldsymbol{g} \cdot \boldsymbol{h})(\boldsymbol{f} \cdot \boldsymbol{n})\right|_{\partial \Omega}=0$. On the other hand, (6) yields

$$
\frac{1}{2} \frac{\mathrm{d}}{\mathrm{d} t}\left\|\boldsymbol{B}^{n}\right\|^{2}=\left(\left(\boldsymbol{B}^{n} \cdot \nabla\left(\boldsymbol{v}^{n}+\boldsymbol{v}_{t}^{n}\right), \boldsymbol{B}^{n}\right)\right) .
$$


From these equalities and $\left(\left(\boldsymbol{v}^{n} \cdot \nabla \boldsymbol{v}^{n}, \boldsymbol{v}_{t}^{n}\right)\right)=\left(\left(\left(\nabla \times \boldsymbol{v}^{n}\right) \times \boldsymbol{v}^{n}, \boldsymbol{v}_{t}^{n}\right)\right)$, we derive

$$
\frac{1}{2} \frac{\mathrm{d}}{\mathrm{d} t}\left(\left\|\boldsymbol{v}^{n}\right\|^{2}+\left\|\nabla \times \boldsymbol{v}^{n}\right\|^{2}+\left\|\boldsymbol{B}^{n}\right\|^{2}\right)=-\left\|\boldsymbol{v}_{t}^{n}\right\|^{2}-\left\|\nabla \times \boldsymbol{v}^{n}\right\|^{2},
$$

which yields

$$
\begin{aligned}
\left\|\boldsymbol{v}^{n}\right\|^{2}+\left\|\boldsymbol{\nabla} \times \boldsymbol{v}^{n}\right\|^{2} & +\left\|\boldsymbol{B}^{n}\right\|^{2}+2 \int_{0}^{t}\left(\left\|\boldsymbol{v}_{t}^{n}\right\|^{2}+\left\|\boldsymbol{\nabla} \times \boldsymbol{v}^{n}\right\|^{2}\right) \mathrm{d} t \\
& =\left\|\left.\boldsymbol{v}^{n}\right|_{t=0}\right\|^{2}+\left\|\nabla \times\left.\boldsymbol{v}^{n}\right|_{t=0}\right\|^{2}+\left\|\left.\boldsymbol{B}^{n}\right|_{t=0}\right\|^{2} \\
& \leq\left\|\boldsymbol{v}_{0}\right\|^{2}+\left\|\nabla \times \boldsymbol{v}_{0}\right\|^{2}+\left\|\boldsymbol{B}_{0}\right\|^{2} .
\end{aligned}
$$

Clearly, this means that the problem (5)-(7) has a unique smooth solution globally in time. Furthermore, using Rellich's theorem, we have the existence of a sequence $\left\{n_{m} \in \mathbb{N} \mid n_{m}<n_{m+1}(m=1,2,3, \ldots)\right\}$ such that $\boldsymbol{v}^{n_{m}}$ converges strongly in $\boldsymbol{H}_{\sigma}$ and weakly in $\boldsymbol{H}_{\sigma}^{1}$, uniformly on an arbitrary countable set of $t$. Since (12) yields the square integrability of $\left\|\nabla \times \boldsymbol{v}^{n}\right\|$ and $\left\|\boldsymbol{v}_{t}^{n}\right\|$ over $(0, \infty)$, we also have the existence of a sequence, for example $\left\{t_{m} \mid 2^{m-1}<t_{m}<2^{m}(m=1,2,3, \ldots)\right\}$, such that

$$
\left\|\nabla \times\left.\boldsymbol{v}^{n_{m}}\right|_{t=t_{m}}\right\|^{2}+\left\|\left.\boldsymbol{v}_{t}^{n_{m}}\right|_{t=t_{m}}\right\|^{2} \leq \frac{1}{2^{m}}\left(\left\|\boldsymbol{v}_{0}\right\|^{2}+\left\|\boldsymbol{\nabla} \times \boldsymbol{v}_{0}\right\|^{2}+\left\|\boldsymbol{B}_{0}\right\|^{2}\right),
$$

that is,

$$
\left\|\nabla \times\left.\boldsymbol{v}^{n_{m}}\right|_{t=t_{m}}\right\| \rightarrow 0, \quad\left\|\left.\boldsymbol{v}^{n_{m}}\right|_{t=t_{m}}\right\| \rightarrow 0, \quad\left\|\left.\boldsymbol{v}_{t}^{n_{m}}\right|_{t=t_{m}}\right\| \rightarrow 0
$$

as $m \rightarrow \infty$. Indeed, if we suppose the nonexistence of this $\left\{t_{m}\right\}$, then we have

$$
\int_{2^{m-1}}^{2^{m}}\left(\left\|\nabla \times \boldsymbol{v}^{n_{m}}\right\|^{2}+\left\|\boldsymbol{v}_{t}^{n_{m}}\right\|^{2}\right) \mathrm{d} t>\frac{1}{2}\left(\left\|\boldsymbol{v}_{0}\right\|^{2}+\left\|\boldsymbol{\nabla} \times \boldsymbol{v}_{0}\right\|^{2}+\left\|\boldsymbol{B}_{0}\right\|^{2}\right),
$$

which conflicts with (12).

Let $\boldsymbol{\Phi} \in \boldsymbol{C}_{0, \sigma}^{\infty}(\Omega)$ be an arbitrary function and define $\boldsymbol{\Phi}^{n} \in \boldsymbol{H}_{\sigma}^{1} \cap \boldsymbol{W}_{2}^{3}(\Omega)$ by

$$
\boldsymbol{\Phi}^{n}=\sum_{j=1}^{n}\left(\left(\boldsymbol{\Phi}, \boldsymbol{w}_{1}^{j}\right)\right) \boldsymbol{w}_{1}^{j}
$$

Then, summing up the products of (5) and $\left(\left(\boldsymbol{\Phi}, \boldsymbol{w}_{1}^{k}\right)\right)$ from $k=1$ to $n$, we obtain

$$
\begin{aligned}
\left(\left(\boldsymbol{v}_{t}^{n}+\left(\boldsymbol{\nabla} \times \boldsymbol{v}^{n}\right) \times \boldsymbol{v}_{t}^{n}, \boldsymbol{\Phi}^{n}\right)\right)= & -\int_{\Omega} \nabla \boldsymbol{\Phi}: \boldsymbol{B}^{n} \otimes \boldsymbol{B}^{n} \mathrm{~d} \boldsymbol{x}+\int_{\Omega} \nabla\left(\boldsymbol{\Phi}-\boldsymbol{\Phi}^{n}\right): \boldsymbol{B}^{n} \otimes \boldsymbol{B}^{n} \mathrm{~d} \boldsymbol{x} \\
& +\left(\left(\boldsymbol{v}^{n} \cdot \nabla \boldsymbol{\Phi}^{n}, \boldsymbol{v}^{n}\right)\right)+\left(\left(\boldsymbol{v}^{n}, \Delta \boldsymbol{\Phi}^{n}\right)\right) .
\end{aligned}
$$

By (12) and the stationary version of [7, Theorem 1], we have the weak-* convergence of $\left.\left|\boldsymbol{B}^{n_{m}}\right|_{t=t_{m}}\right|^{2}$ to a measure $\mu$ in $M(\Omega)$, which yields (8), and the limit

$$
\lim _{m \rightarrow \infty} \int_{\Omega} \nabla \boldsymbol{\Phi}:\left.\left(\boldsymbol{B}^{n_{m}} \otimes \boldsymbol{B}^{n_{m}}\right)\right|_{t=t_{m}} \mathrm{~d} \boldsymbol{x}=\int_{\Omega} \boldsymbol{\nabla} \boldsymbol{\Phi}:\left(\int_{S^{2}} \frac{\boldsymbol{u}}{|\boldsymbol{u}|} \otimes \frac{\boldsymbol{u}}{|\boldsymbol{u}|} \mathrm{d} \nu_{2, \boldsymbol{x}}\right) \mathrm{d} \mu
$$


with a $\mu$-measurable $\nu_{2, .}: \Omega \rightarrow \operatorname{Prob} M\left(S^{2}\right)$. Since

$$
\sum_{i=1}^{3} \sup _{\left(x_{1}, x_{2}, x_{3}\right) \in \Omega}\left|\frac{\partial \Phi}{\partial x_{i}}-\frac{\partial \Phi^{n}}{\partial x_{i}}\right| \leq \text { const. }\left\|\boldsymbol{\Phi}-\Phi^{n}\right\|_{3} \quad(\rightarrow 0 \text { as } n \rightarrow \infty)
$$

follows from Sobolev's embedding theorem and [13, Chapter 2, Theorem 7], we derive (10) by using (13) in (14).

From the equality $\left.\left(\nabla \phi, \boldsymbol{B}^{n}\right)\right)=0$ for any $\phi \in C_{0}^{\infty}(\Omega)$, we deduce (11) by the stationary version of [7, Theorem 1].

Lastly, let us prove $|\mu|_{M(\Omega)}>0$ under (9). Multiplying (6) by $\lambda_{2, k}^{-1} b_{n, k}$ and summing up the products from $k=1$ to $n$, we have

$$
\begin{aligned}
\frac{1}{2} \frac{\mathrm{d}}{\mathrm{d} t}\left(\left(\boldsymbol{B}^{n}, A \boldsymbol{B}^{n}\right)\right) & =\frac{1}{2} \frac{\mathrm{d}}{\mathrm{d} t} \sum_{k=1}^{n} \lambda_{2, k}^{-1} b_{n, k}^{2} \\
& =\sum_{k=1}^{n}\left(\left(\nabla \times\left(\left(\boldsymbol{v}^{n}+\boldsymbol{v}_{t}^{n}\right) \times \boldsymbol{B}^{n}\right), \lambda_{2, k}^{-1} b_{n, k} \boldsymbol{w}_{2}^{k}\right)\right) \\
& =\sum_{k=1}^{n}\left(\left(\left(\boldsymbol{v}^{n}+\boldsymbol{v}_{t}^{n}\right) \times \boldsymbol{B}^{n}, b_{n, k} \boldsymbol{w}_{2}^{k}\right)\right)=0 .
\end{aligned}
$$

Here $A=(\nabla \times)^{-1}: \boldsymbol{H}_{\sigma} \rightarrow\left\{\boldsymbol{f} \in \boldsymbol{H}_{\sigma} \mid \nabla \times \boldsymbol{f} \in \boldsymbol{H}_{\sigma}\right\}$ (see [23]). Therefore,

$$
\left(\left(\boldsymbol{B}^{n}, A \boldsymbol{B}^{n}\right)\right)=\left.\left(\left(\boldsymbol{B}^{n}, A \boldsymbol{B}^{n}\right)\right)\right|_{t=0} \rightarrow\left(\left(\boldsymbol{B}_{0}, A \boldsymbol{B}_{0}\right)\right)=\sum_{j=1}^{\infty} \lambda_{2, j}^{-1}\left(\left(\boldsymbol{B}_{0}, \boldsymbol{w}_{2}^{j}\right)\right)^{2} \neq 0
$$

as $n \rightarrow \infty$. Now, suppose that $|\mu|_{M(\Omega)}=0$, that is, $\left.\lim _{m \rightarrow \infty} \int_{\Omega}\left|\boldsymbol{B}^{n_{m}}\right|_{t=t_{m}}\right|^{2} \phi \mathrm{d} \boldsymbol{x}=0$ is valid for any $\phi \in C_{0}^{\infty}(\Omega)$. Then, since

$$
\left\|A \boldsymbol{B}^{n}\right\|_{1}^{2} \leq C\left\|\boldsymbol{B}^{n}\right\|^{2} \leq C\left(\left\|\boldsymbol{v}_{0}\right\|^{2}+\left\|\boldsymbol{\nabla} \times \boldsymbol{v}_{0}\right\|^{2}+\left\|\boldsymbol{B}_{0}\right\|^{2}\right)
$$

is obtained, where $C$ is a positive constant independent of $n$, and

$$
\begin{aligned}
\left|\left(\left(\left.A \boldsymbol{B}^{n_{m}}\right|_{t=t_{m}}, \nabla \times \boldsymbol{\Phi}\right)\right)\right| & =\left|\left(\left(\left.\boldsymbol{B}^{n_{m}}\right|_{t=t_{m}}, \boldsymbol{\Phi}\right)\right)\right| \\
& \leq\left(\left.\int_{\Omega} \mathrm{d} \boldsymbol{x} \int_{\Omega}\left|\boldsymbol{B}^{n_{m}}\right|_{t=t_{m}}\right|^{2}|\boldsymbol{\Phi}|^{2} \mathrm{~d} \boldsymbol{x}\right)^{1 / 2}(\rightarrow 0 \text { as } m \rightarrow \infty)
\end{aligned}
$$

holds for any $\boldsymbol{\Phi} \in \boldsymbol{C}_{0, \sigma}^{\infty}(\Omega)$, we have the convergence $\left.A \boldsymbol{B}^{n_{m}}\right|_{t=t_{m}} \rightarrow \mathbf{0}$ strongly in $\boldsymbol{H}_{\sigma}$. It means that $\left.\left(\left(\boldsymbol{B}^{n_{m}}, A \boldsymbol{B}^{n_{m}}\right)\right)\right|_{t=t_{m}} \rightarrow 0$, which conflicts with (16). Hence $|\mu|_{M(\Omega)}>0$ is deduced.

Remark. As was mentioned in Section 2, the function $c \boldsymbol{w}_{2}^{k}(k \in \mathbb{N})$ with an arbitrary nonzero constant $c$ satisfies (1) with $\left.\boldsymbol{u} \cdot \boldsymbol{n}\right|_{\partial \Omega}=0$. It can be regarded as a measure-valued solution:

$$
\mathrm{d} \mu=c^{2}\left|\boldsymbol{w}_{2}^{k}\right|^{2} \mathrm{~d} \boldsymbol{x}, \quad v_{1, \boldsymbol{x}}=\delta_{c w_{2}^{k}(\boldsymbol{x})}, \quad v_{2, \boldsymbol{x}}=\delta_{c \boldsymbol{w}_{2}^{k}(\boldsymbol{x}) /\left|c \boldsymbol{w}_{2}^{k}(\boldsymbol{x})\right|},
$$


where $\delta_{f}$ is the Dirac measure at $f$. It is open whether or not our Theorem really means the existence of a measure-valued solution which is different from (17).

In the two-dimensional case, which will be discussed in another paper, we can obtain an analogous result to the Theorem by choosing another set of smooth solutions to (1) as $\left\{\boldsymbol{w}_{2}^{k}\right\}_{k=1}^{\infty}$.

In [10], Freedman investigated (2) assuming the existence of a solution on a closed 3-manifold. He proved that a magnetic field with a nontrivial link has a positive lower bound of energy even if the magnetic helicity is equal to zero. The condition (9) in our theorem means that $\boldsymbol{B}_{0}$ has a nonzero magnetic helicity. It is open whether or not we can obtain $|\mu|_{M(\Omega)}>0$ without (9) by applying Freedman's theory.

Acknowledgement. The author thanks Professor Tetsuro Miyakawa for his information about [23].

\section{REFERENCES}

1. H. D. Alber, Existence of three dimensional, steady, inviscid, incompressible flows with nonvanishing vorticity, Math. Ann. 292 (1992), 493-528.

2. A. Ambrosetti and M. Struwe, Existence of steady vortex rings in an ideal fluid, Arch. Rational Mech. Anal. 108 (1989), 97-109.

3. T. V. Badiani, Existence of steady symmetric vortex pairs on a planar domain with an obstacle, Math. Proc. Cambridge Philos. Soc. 123 (1998), 365-384.

4. T. Z. Boulmezaoud and T. Amari, On the existence of non-linear force-free fields in three-dimensional domains, Z. Angew. Math. Phys. 51 (2000), 942-967.

5. G. R. Burton, Steady symmetric vortex pairs and rearrangements, Proc. Roy. Soc. Edinburgh Sect. A 108 (1988), 269-290.

6. D. Chae, The vanishing viscosity limit of statistical solutions of the Navier-Stokes equations. II. The general case, J. Math. Anal. Appl. 155 (1991), 460-484.

7. R. J. DiPerna and A. J. Majda, Oscillations and concentrations in weak solutions of the incompressible fluid equations, Comm. Math. Phys. 108 (1987), 667-689.

8. R. J. DiPerna and A. J. Majda, Concentrations in regularizations for 2-D incompressible flow, Comm. Pure Appl. Math. 40 (1987), 301-345.

9. L. E. Fraenkel and M. S. Berger, A global theory of steady vortex rings in an ideal fluid, Acta Math. 132 (1974), 13-51.

10. M. H. Freedman, A note on topology and magnetic energy in incompressible perfectly conducting fluids, J. Fluid Mech. 194 (1988), 549-551.

11. A. Friedman and B. Turkington, Vortex rings: existence and asymptotic estimates, Trans. Amer. Math. Soc. 268 (1981), 1-37.

12. R. Kaiser, M. Neudert and W. von Wahl, On the existence of force-free magnetic fields with small nonconstant $\alpha$ in exterior domains, Comm. Math. Phys. 211 (2000), 111-136.

13. O. A. Ladyzhenskaya, The Mathematical Theory of Viscous Incompressible Flow (Gordon and Breach, 1969).

14. H. K. Moffatt, Magnetostatic equilibria and analogous Euler flows of arbitrarily complex topology. Part 1. Fundamentals, J. Fluid Mech. 159 (1985), 359-378.

15. H. K. Moffatt, Structure and stability of solutions of the Euler equations: a lagrangian approach, Philos. Trans. Roy. Soc. London Ser. A 333 (1990), 321-342. 247.

16. W.-M. Ni, On the existence of global vortex rings, J. d'Analyse Math. 37 (1980), 208-

17. T. Nishiyama, Pseudo-advection method for the two-dimensional stationary Euler equations, Proc. Amer. Math. Soc. 129 (2001), 429-432.

18. O. V. Troshkin, A two-dimensional flow problem for steady-state Euler equations, Math. USSR Sbornik 66 (1990), 363-382.

19. B. Turkington, On steady vortex flow in two dimensions, Comm. Partial Differential Equations 8 (1983), 999-1030, 1031-1071. 
20. B. Turkington, Vortex rings with swirl: axisymmetric solutions of the Euler equations with nonzero helicity, SIAM J. Math. Anal. 20 (1989), 57-73.

21. G. K. Vallis, G. F. Carnevale and W. R. Young, Extremal energy properties and construction of stable solutions of the Euler equations, J. Fluid Mech. 207 (1989), 133-152.

22. G. Wolansky, Existence, uniqueness, and stability of stationary barotropic flow with forcing and dissipation, Comm. Pure Appl. Math. 41 (1988), $19-46$.

23. Z. Yoshida and Y. Giga, Remarks on spectra of operator rot, Math. Z. 204 (1990), $235-245$ 